\title{
Association of thyroid carcinoma with pregnancy: A meta-analysis
}

\author{
Y.Q. ZHOU, Z.ZHOU, M.F. QIAN, T. GONG and J.D. WANG \\ Department of Head and Neck Surgery, Ren Ji Hospital, \\ Shanghai Jiaotong University School of Medicine, Shanghai 200127, P.R. China
}

Received June 9, 2014; Accepted September 12, 2014

DOI: $10.3892 / \mathrm{mco} .2014 .472$

\begin{abstract}
A number of scholars reported that reproductive factors play a significant role in thyroid cancer and the correlation between the two may affect the diagnosis and treatment of thyroid carcinoma during pregnancy. To determine whether pregnancy reproductive factors affect thyroid carcinoma, we conducted a meta-analysis of studies that investigated the association between pregnancy factors and thyroid carcinoma. PubMed, OVID and the Cochrane Library were searched from their inception to April 1st, 2013. The searched publications mainly investigated reproductive factors and the morbidity or prognosis of female thyroid carcinoma. The studies were filtered by predetermined standards and the quality of the included studies was evaluated by the Newcastle-Ottawa scale inventory. Two researchers independently extracted information on first author, year of publication, study design (case-control or prospective cohort), compared populations, inclusion and exclusion criteria and total sample size. Other researchers assessed the studies for publication bias and performed statistical analyses. Discrepancies were resolved by consensus. A total of 21 studies were selected for the meta-analysis, including 406,329 cases in total. Compared to the control group, the risk of thyroid carcinoma in women with a history of pregnancy was not significantly discrepant, [odds ratio $(\mathrm{OR})=1.00,95 \%$ confidence interval $(\mathrm{CI})$ : 0.91-1.11]. However, the risk of thyroid carcinoma in women with a history of $\geq 3$ pregnancies was significantly increased (OR=1.39, 95\% CI: 1.21-1.59). Furthermore, an interval of $\leq 5$ years since the last pregnancy was closely associated with thyroid carcinoma $(\mathrm{OR}=1.53,95 \% \mathrm{CI}: 1.29-1.81)$. The patients developed thyroid carcinoma during pregnancy did not exhibit an increased risk of lymphatic metastasis $(\mathrm{OR}=0.94$, 95\% CI: 0.53-1.67); the risk of distant metastasis also did not increase significantly $(\mathrm{OR}=1.03,95 \% \mathrm{CI}$ : 0.86-1.24). Therefore, multiple pregnancies and a $\leq 5$-year interval between pregnancies were identified as high-risk factors for thyroid carcinoma,
\end{abstract}

Correspondence to: Dr J.D. Wang, Department of Head and Neck Surgery, Ren Ji Hospital, Shanghai Jiaotong University School of Medicine, 145 Middle Shandong Road, Shanghai 200127, P.R. China E-mail: drjiadongwang@163.com

Key words: pregnancy, thyroid neoplasm, reproduction, meta-analysis whereas thyroid carcinoma during pregnancy was not associated with a significant risk of lymphatic and distant metastasis.

\section{Introduction}

The association between pregnancy and thyroid carcinoma has been debated upon for over half a century; however, no definitive conclusion has been reached. Differentiated thyroid cancer ranks second in incidence among tumors diagnosed during pregnancy, affecting 14/100,000 individuals (1). Over the last few decades, a number of scholars indicated that reproductive factors played a significant role in thyroid cancer. Certain investigators considered that the risk of thyroid carcinoma increased with an increasing number of births (2). Moreover, thyroid carcinoma during pregnancy was found to be more aggressive and its prognosis was worse following radical resection, whereas thyroid carcinoma during pregnancy was a predictor of recurrence, which was considered to be associated with hormone receptors and hormone level fluctuations (3). However, other scholars reported that the development and progression of thyroid carcinoma were not significantly associated with pregnency. Negri et al (4) conducted a meta-analysis in 1999 investigating the correlation between reproductive factors and thyroid carcinoma. That study indicated a weak correlation, whereas the reproductive factors in young women were significantly associated with thyroid carcinoma. Decades later, following the publication of numerous large-scale clinical trials, this remains a controversial subject. Therefore, we performed this meta-analysis to investigate the association between reproductive factors and thyroid carcinoma.

\section{Materials and methods}

Study selection. We followed the guidelines of Meta-analysis of Observational Studies in Epidemiology (5) to conduct a meta-analysis in order to determine the association between thyroid carcinoma and pregnancy. The search terms used were pregnancy, reproduction and thyroid neoplasms. Three main databases, namely PubMed, OVID and the Cochrane Library, were searched from their inception to April 1st, 2013. The search strategies were medical subject headings combined with key words. To ensure a comprehensive search, we also searched the reference lists of the included studies and previously published reviews. The authors were contacted when data were ambiguous or missing. Subsequently, the abstracts were screened and the full-text articles were accessed. No language 
restriction was applied. The study selection process is summarized in Fig. 1.

Inclusion criteria. The original studies were prospective or retrospective random case-control trials; except for the investigated factors, there was no other difference between the experimental and control groups. All the diagnoses were pathologically confirmed, pregnancy was diagnosed by ultrasound and the follow-up period in all the cases was $>6$ months.

Exclusion criteria. We excluded reviews, editorials, letters, case series, case reports and conference proceedings; studies other that random case-control trials; studies with different diagnostic standards and inappropriate outcome measurements; and studies that provided no original data or only unadjusted analysis. Studies with limited follow-up period were also eliminated.

Data extraction and quality assessment. Two investigators extracted data from the eligible studies. The data included first author, year of publication, study design (case-control or prospective cohort), compared populations, inclusion and exclusion criteria, total sample size, number of patients in the thyroid carcinoma and control groups and number of pregnant individuals in each group. We evaluated and filtered all the eligible clinical studies, grading each study according to the Newcastle-Ottawa scale (NOS) (6), with 0-4 points reflecting low quality and 5-9 high quality. Any disagreements or discrepancies were resolved by consensus.

Statistical analysis. We classified the selected studies, extracted data and performed a meta-analysis using RevMan 5.1 software (http://tech.cochrane.org/revman/download), which was provided by the Cochrane Library.

\section{Results}

Search results and basic characteristics of eligible studies. Of the 627 retrieved articles, 39 abstracts were selected for full-text screening, including 1 Chinese study written in English. The inclusion criteria were met by 21 of the 39 studies (Fig. 1). According to the exclusion criteria, 18 studies were eliminated (3 studies reported the effect of pregnancy after surgery, 2 focused on the association between thyroid nodules and pregnancy, 1 used fine needle aspiration as the diagnostic method, 1 had a different research object, 2 were meta-analyses, 1 had no control group, 4 focused on surgical treatment, 2 had insufficient samples and 2 did not provide original data). The 21 selected studies included 406,329 cases in total (Table I).

Association between thyroid carcinoma and reproductive factors

Association between thyroid carcinoma and pregnancy history. A total of 17 studies, including 402,402 cases, investigated the association between pregnancy and thyroid cancer. Of those 17 studies, 2 were prospective studies of high quality. A proportion of the studies did not include detailed information, such as the number of pregnancies or the age at pregnancy. As there was no heterogeneity $(\mathrm{P}=0.18)$, the fixed-effects model

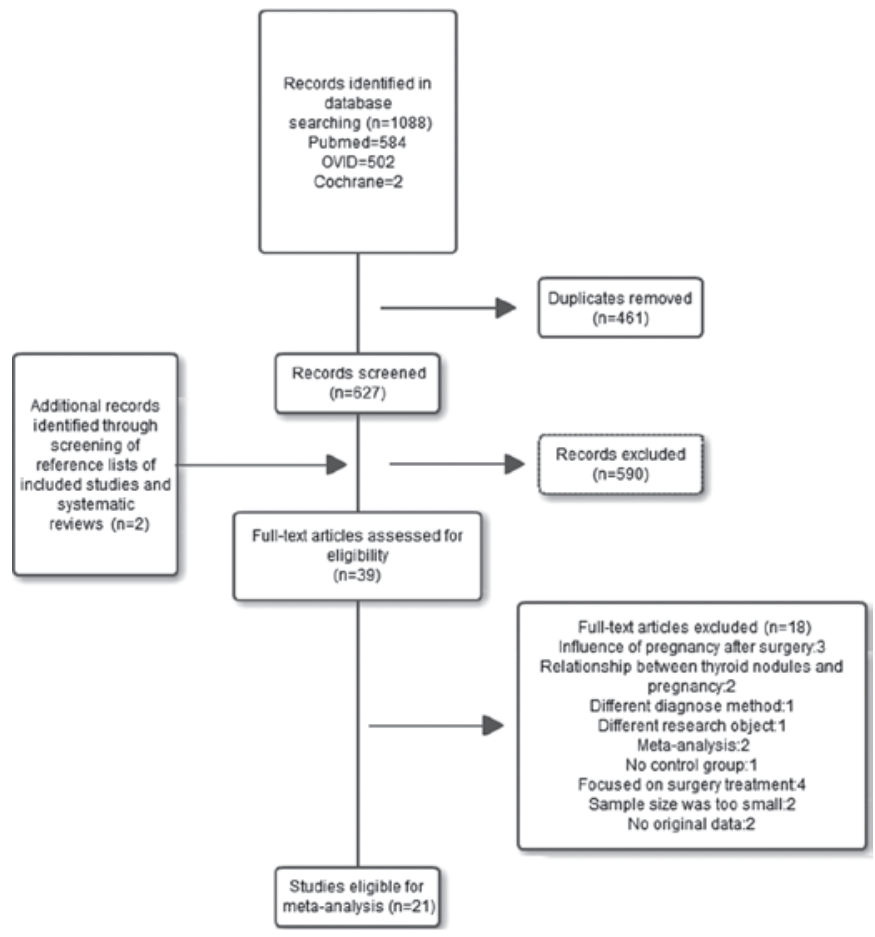

Figure 1. Flowchart of the study selection process.

was selected. A combined analysis of the 17 studies [odds ratio $(\mathrm{OR})=1.00,95 \%$ confidence interval $(\mathrm{CI})$ : 0.91-1.11] revealed that pregnancy history is not significantly associated with the risk of thyroid carcinoma (Fig. 2).

Association between thyroid carcinoma and $\geq 3$ pregnancies. Following a precision screening, 8 studies were included. Of those 8 studies, 2 indicated that $\geq 3$ pregnancies was a risk factor for thyroid cancer, while the remaining 6 studies reported opposing results. There were 4,553 cases in total, without obvious heterogeneity $(\mathrm{P}=0.11)$; therefore, the fixed-effects model was selected. In a combined analysis of all 8 studies $(\mathrm{OR}=1.39,95 \% \mathrm{CI}: 1.21-1.59)$, the results demonstrated that women with $\geq 3$ pregnancies exhibited an increased risk for thyroid carcinoma (Fig. 3).

Association between thyroid carcinoma and an interval of $\leq 5$ years since the last pregnancy. Five studies were included, with a total of 120,278 cases. There was no heterogeneity $(\mathrm{P}=0.76)$, so the fixed-effects model was selected. In a combined analysis of all 5 studies (OR=1.53,95\% CI: 1.29-1.81), we observed that, compared to the control group, more women in the thyroid carcinoma group had become pregnant within 5 years since their last pregnancy (Fig. 4). Therefore, an interval of $\leq 5$ years since the last pregnancy is associated with a higher risk for thyroid carcinoma.

\section{Effect of pregnancy on lymph node and distant metastasis} Thyroid carcinoma during pregnancy and distant metastasis. A total of 3 studies were included in the analysis of the association between thyroid carcinoma during pregnancy and distant metastasis. There were 671 cases in the experimental and 2,859 cases in the control group. There was no heterogeneity $(\mathrm{P}=0.27)$ and the fixed-effects model was used $(\mathrm{OR}=0.94$, 95\% CI: 0.53-1.67) (Fig. 5). The results indicated that pregnancy did not increase the distant metastasis rate. 
Table I. Basic characteristics of eligible studies.

\begin{tabular}{|c|c|c|c|c|c|c|c|}
\hline Authors & $\begin{array}{c}\text { Year of } \\
\text { publication }\end{array}$ & $\begin{array}{l}\text { Study } \\
\text { design }\end{array}$ & Population & Cases & Controls & NOS & (Refs.) \\
\hline Akslen et al & 1992 & Prospective cohort & Norway & 124 & 62,966 & 8 & (7) \\
\hline Brindel et al & 2008 & Case-control & France & 201 & 324 & 7 & (2) \\
\hline Galanti et al & 1996 & Case-control & Norway-Sweden & 191 & 341 & 7 & (8) \\
\hline Hallquist et al & 1994 & Case-control & Sweden & 180 & 360 & 6 & (9) \\
\hline Horn-Ross et al & 2011 & Case-control & USA & 233 & 117,413 & 7 & $(10)$ \\
\hline Kolonel et al & 1990 & Case-control & USA & 140 & 328 & 7 & (11) \\
\hline Levi et al & 1993 & Case-control & Switzerland & 91 & 306 & 6 & (12) \\
\hline Mack et al & 1999 & Case-control & USA & 292 & 292 & 5 & (13) \\
\hline McTiernan et al & 1984 & Case-control & USA & 185 & 359 & 6 & (14) \\
\hline Memon et al & 2002 & Case-control & Kuwait & 238 & 238 & 8 & $(15)$ \\
\hline Moosa and Mazzaferri & 1997 & Case-control & USA & 61 & 528 & 6 & (16) \\
\hline Preston-Martin et al & 1987 & Case-control & USA & 292 & 292 & 5 & $(17)$ \\
\hline Preston-Martin et al & 1993 & Case-control & China & 207 & 207 & 5 & $(18)$ \\
\hline Rossing et al & 2000 & Case-control & USA & 410 & 574 & 6 & (19) \\
\hline Schonfeld et al & 2011 & Prospective cohort & USA & 312 & 187,553 & 8 & $(20)$ \\
\hline Takezaki et al & 1996 & Case-control & Japan & 94 & 26,666 & 5 & $(21)$ \\
\hline Truong et al & 2005 & Case-control & France & 293 & 354 & 9 & $(22)$ \\
\hline Vannucchi et al & 2010 & Case-control & England & 15 & 61 & 7 & (3) \\
\hline Wingren et al & 1993 & Case-control & Sweden & 132 & 203 & 7 & (23) \\
\hline Yasmeen et al & 2005 & Case-control & USA & 595 & 2,270 & 7 & (24) \\
\hline Zivaljevic et al & 2003 & Case-control & Serbia & 204 & 204 & 6 & $(25)$ \\
\hline
\end{tabular}

NOS, Newcastle-Ottawa scale.

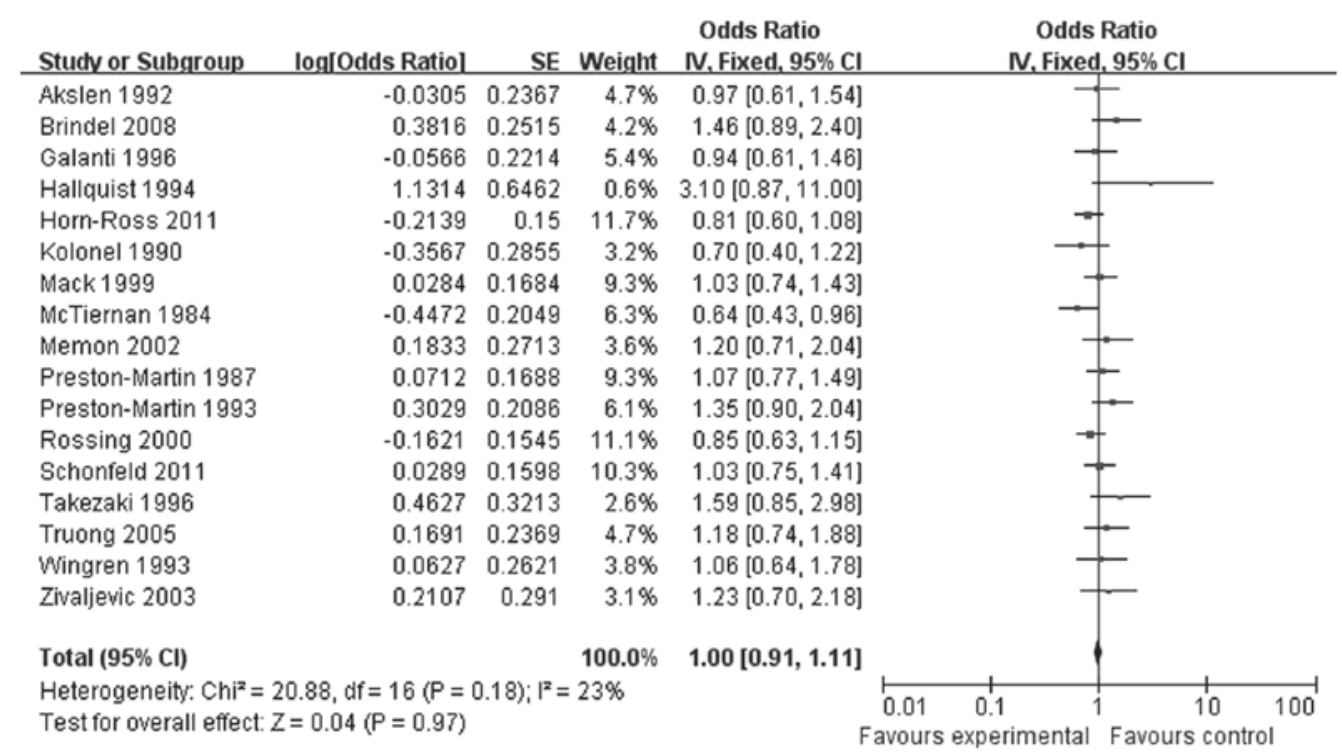

Figure 2. Meta-analysis of the association between thyroid carcinoma and pregnancy history. Total odds ratio = 1.00 (95\% CI: $0.91-1.11)$, indicating that pregnancy history was not significantly associated with the risk for thyroid carcinoma. SE, standard error; CI, confidence interval.

Thyroid carcinoma during pregnancy and lymphatic metastasis. A total of 3 studies were included in the analysis of the association between pregnancy and lymphatic metastasis of thyroid carcinoma (Fig. 6). There were 671 cases in the experimental and 2,859 cases in the control group. There was no significant heterogeneity $(\mathrm{P}=0.18)$ and the fixed-effects model was used ( $\mathrm{OR}=1.03,95 \% \mathrm{CI}$ : 0.86-1.24). The results indicated that pregnancy did not increase the rate of lymphatic metastasis.

Publication bias and sensitivity analysis. We used a funnel graph to represent publication bias and 17 studies that investigated the association between thyroid carcinoma and 


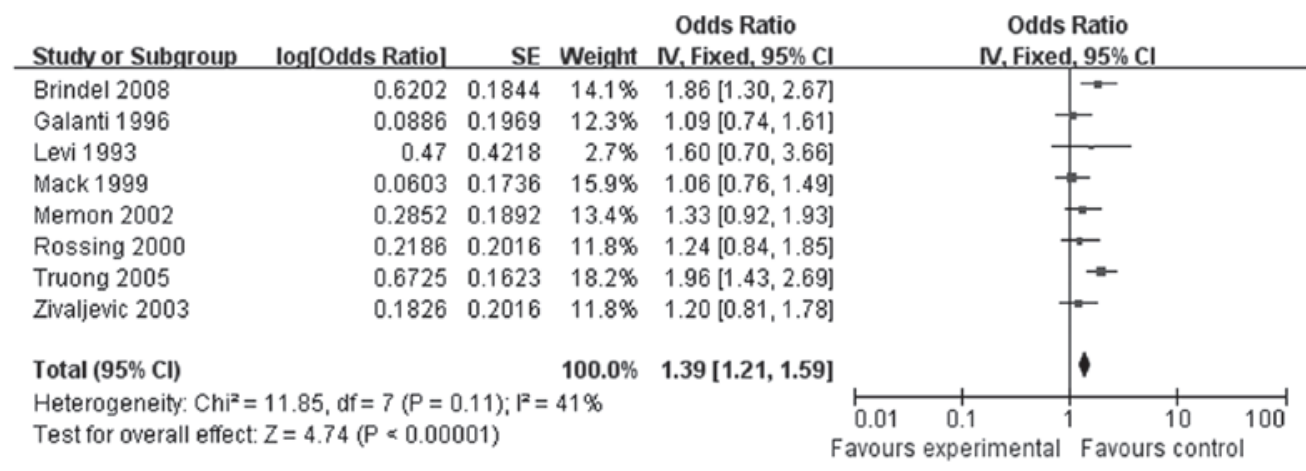

Figure 3. Meta-analysis of the association between thyroid carcinoma and $\geq 3$ pregnancies. Total odds ratio $=1.39$ (95\% CI: $1.21-1.59)$, indicating that women with $\geq 3$ pregnancies were at higher risk for thyroid carcinoma. SE, standard error; CI, confidence interval.

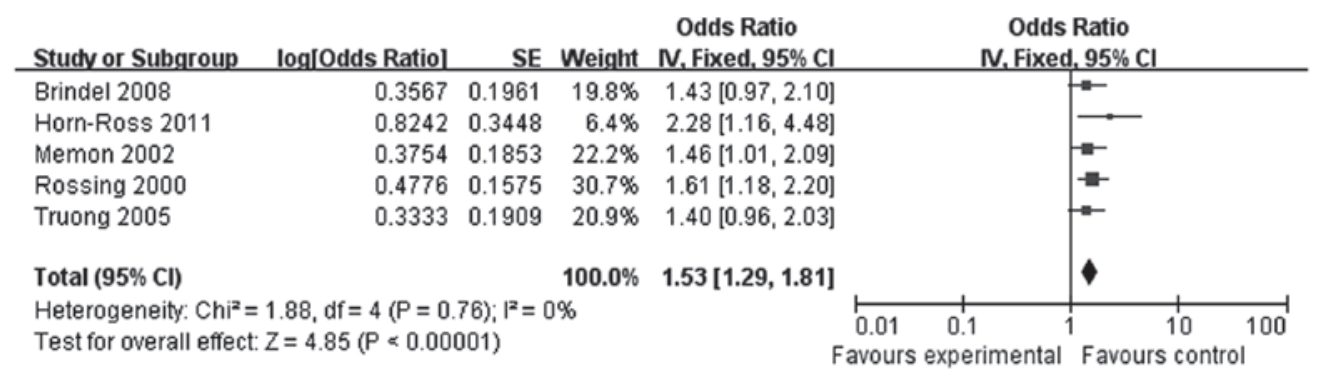

Figure 4. Meta-analysis of the association between thyroid carcinoma and an interval of $\leq 5$ years since the last pregnancy. Total odds ratio $=1.53$ (95\% CI: 1.29-1.81), indicating that an interval of $\leq 5$ years since the last pregnancy is associated with a higher risk for thyroid carcinoma. SE, standard error; CI, confidence interval.

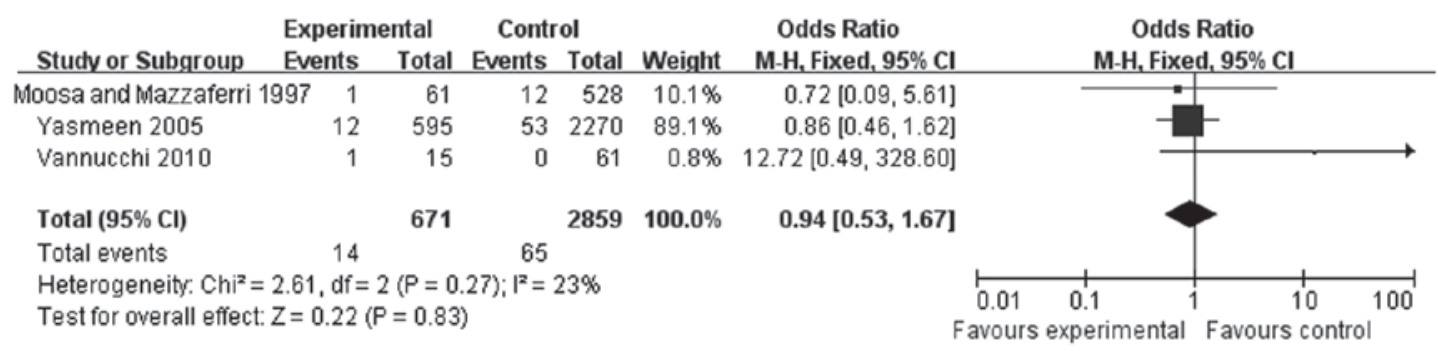

Figure 5. Meta-analysis of the association between thyroid carcinoma during pregnancy and distant metastasis. Total odds ratio $=0.94$ (95\% CI: $0.53-1.67$ ), indicating that pregnancy did not increase the distant metastasis rate. CI, confidence interval.

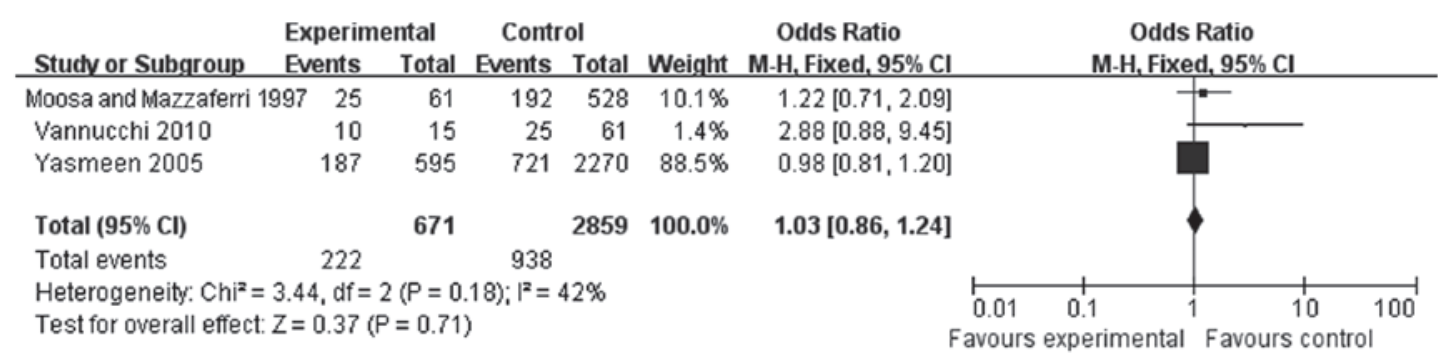

Figure 6. Meta-analysis of the association between thyroid carcinoma during pregnancy and lymphatic metastasis. Total odds ratio=1.03 (95\% CI: 0.86-1.24), indicating that pregnancy did not increase the lymphatic metastasis rate. CI, confidence interval.

pregnancy history did not exhibit publication bias (Fig. 7). There was no significant heterogeneity in the analysis of thyroid carcinoma during pregnancy and lymphatic metastasis, but the heterogeneity was significant, so the sensitivity was analyzed. The effect of each study on the combined estimate was assessed by removing one study at a time, which did not affect the significance of the combined estimate or heterogeneity. It appeared that the heterogeneity originated from the study by Vannucchi et al (3), which included a relatively limited number of cases. 


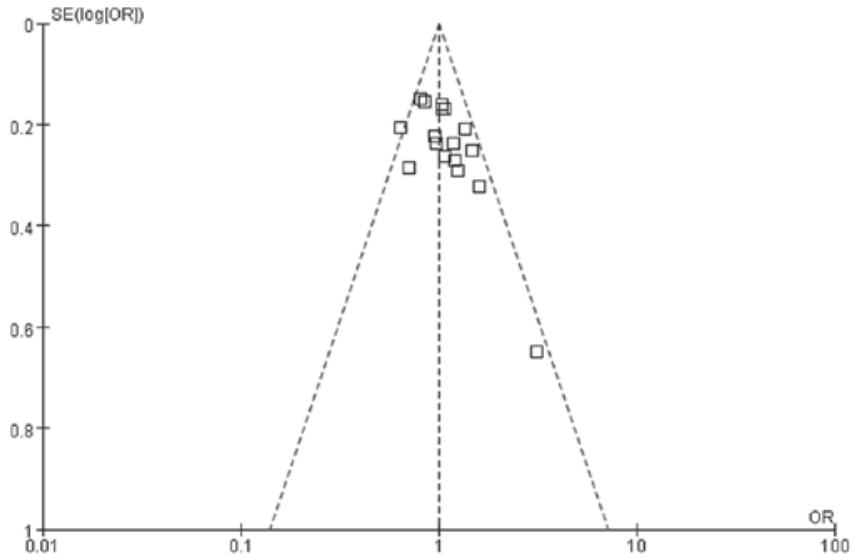

Figure 7. Funnel plot for the assessment of 17 studies investigating the association between thyroid carcinoma and pregnancy history. The funnel graph indicates that there was no publication bias among the studies on the association between thyroid carcinoma and pregnancy history. SE, standard error; OR, odds ratio.

\section{Discussion}

Our study suggested a significant association between pregnancy and thyroid carcinoma. The majority of the subjects in the included studies were diagnosed with differentiated thyroid carcinoma. Several investigators have focused on the mechanism underlying this correlation and a likely explanation is hormone level fluctuation. The trophoblast cells secrete human chorionic gonadotropin (hCG) and progesterone during pregnancy, as well as estrogen during late pregnancy, to regulate the delivery process. As hCG is considered to have a similar structure with thyroid-stimulating hormone (TSH), it may combine with the thyroid-stimulating hormone receptor (TSHR). When high concentrations of hCG are secreted into the bloodstream, it may combine with TSHR more than TSH and promote thyroid cell proliferation (26). Estrogen has been proven to promote tumor growth significantly and this effect is achieved by combining with the estrogen receptor (ER). Kumar et al (27) reported that estrogen acts like an ER agonist, which may significantly promote the growth of differentiated thyroid carcinoma. Moreover, estrogen may inhibit apoptosis of thyroid carcinoma cells by increasing the expression of a series of proteins, such as Bcl-2. The role of progesterone in thyroid carcinoma remains controversial. In our meta-analysis, we observed a significant association between a history of multiple pregnancies and thyroid carcinoma. In addition, an interval of $\leq 5$ years since the last pregnancy significantly increased the risk of thyroid carcinoma. These results indicate that hormone level fluctuation increases the risk of thyroid carcinoma.

The data in our study were independently extracted by two investigators. There was no obvious publication bias and no language restriction. The included studies involved a total of 406,329 cases and were of clinical significance. The regions and ethnicity of the studies reflected international diversity, including countries such as China, USA, Britain and Norway. Although our meta-analysis was not the first to investigate the association between pregnancy and thyroid carcinoma, it was an important supplementary study to earlier research. We evaluated all the included studies by the NOS and then identified the studies with significant bias.

There were certain limitations to our study and heterogeneity is a major issue that must be taken into consideration. There was no significant heterogeneity in each analysis, while the analysis of the association between thyroid carcinoma during pregnancy and lymphatic metastasis revealed significant heterogeneity. All the included studies were of high quality. The funnel plot revealed no publication bias, but the evaluating ability of the funnel plot declines when there are fewer studies included. Sensitivity was then analyzed. The heterogeneity appeared to originate from the study of Vannucchi et al (3), which included a relatively limited number of cases. In addition, other factors may affect the incidence or invasive potential of thyroid cancer, such as age, tumor size and the degree of differentiation, but we were unable to assess these factors due to the lack of detailed data.

Although this study has several limitations, as mentioned above, it demonstrated a close association between pregnancy and thyroid carcinoma in certain aspects. In this study, the risk for thyroid carcinoma was not found to be increased during pregnancy, but the limited number of studies and included cases were not sufficient to reach definitive conclusions. Further large prospective clinical trials are required, focusing on thyroid carcinoma during pregnancy, to supplement and update our data and also perform a subgroup analysis according to age, history and pathological types, to draw a more definitive conclusion.

In summary, pregnancy was identified as a risk factor of thyroid carcinoma and multiparous women should be closely followed up to detect the disease early. Thyroid carcinoma during pregnancy does not increase the probability of lymphatic or distant metastasis. Treatment should be individualized and the significance of follow-up must be emphasized.

\section{Acknowledgements}

This study was supported by the Shanghai Municipal Health Bureau (grant no. 2012029).

\section{References}

1. Smith LH,Danielsen B, Allen ME and Cress R: Cancer associated with obstetric delivery: results of linkage with the California cancer registry. Am J Obstet Gynecol 189: 1128-1135, 2003.

2. Brindel P, Doyon F, Rachedi F, et al: Menstrual and reproductive factors in the risk of differentiated thyroid carcinoma in native women in French Polynesia: a population-based case-control study. Am J Epidemiol 167: 219-229, 2008.

3. Vannucchi G, Perrino M, Rossi S, et al: Clinical and molecular features of differentiated thyroid cancer diagnosed during pregnancy. Eur J Endocrinol 162: 145-151, 2010.

4. Negri E, Dal Maso L, Ron E, et al: A pooled analysis of case-control studies of thyroid cancer. II. Menstrual and reproductive factors. Cancer Causes Control 10: 143-155, 1999.

5. Stroup DF, Berlin JA, Morton SC, et al: Meta-analysis of observational studies in epidemiology: a proposal for reporting. Meta-analysis Of Observational Studies in Epidemiology (MOOSE) group. JAMA 283: 2008-2012, 2000.

6. Ownby RL, Crocco E, Acevedo A, John V and Loewenstein D: Depression and risk for Alzheimer disease: systematic review, meta-analysis, and metaregression analysis. Arch Gen Psychiatry 63: 530-538, 2006.

7. Akslen LA, Nilssen S and Kvale G: Reproductive factors and risk of thyroid cancer. A prospective study of 63,090 women from Norway. Br J Cancer 65: 772-774, 1992. 
8. Galanti MR, Hansson L, Lund E, et al: Reproductive history and cigarette smoking as risk factors for thyroid cancer in women: a population-based case-control study. Cancer Epidemiol Biomarkers Prev 5: 425-431, 1996.

9. Hallquist A, Hardell L, Degerman A and Boquist L: Thyroid cancer: reproductive factors, previous diseases, drug intake, family history and diet. A case-control study. Eur J Cancer Prev 3: 481-488, 1994.

10. Horn-Ross PL, Canchola AJ, Ma H, Reynolds P and Bernstein L: Hormonal factors and the risk of papillary thyroid cancer in the California Teachers Study cohort. Cancer Epidemiol Biomarkers Prev 20: 1751-1759, 2011.

11. Kolonel LN, Hankin JH, Wilkens LR, Fukunaga FH and Hinds MW: An epidemiologic study of thyroid cancer in Hawaii. Cancer Causes Control 1: 223-234, 1990.

12. Levi F, Franceschi S, Gulie C, Negri E and La Vecchia C: Female thyroid cancer: the role of reproductive and hormonal factors in Switzerland. Oncology 50: 309-315, 1993.

13. Mack WJ, Preston-Martin S, Bernstein L, Qian D and Xiang M: Reproductive and hormonal risk factors for thyroid cancer in Los Angeles County females. Cancer Epidemiol Biomarkers Prev 8: 991-997, 1999.

14. McTiernan AM, Weiss NS and Daling JR: Incidence of thyroid cancer in women in relation to reproductive and hormonal factors. Am J Epidemiol 120: 423-435, 1984.

15. Memon A, Darif M, Al-Saleh K and Suresh A: Epidemiology of reproductive and hormonal factors in thyroid cancer: evidence from a case-control study in the Middle East. Int J Cancer 97: $82-89,2002$

16. Moosa $M$ and Mazzaferri EL: Outcome of differentiated thyroid cancer diagnosed in pregnant women. J Clin Endocrinol Metab 82: 2862-2866, 1997.

17. Preston-Martin S, Bernstein L, Pike MC, Maldonado AA and Henderson BE: Thyroid cancer among young women related to prior thyroid disease and pregnancy history. $\mathrm{Br} \mathrm{J}$ Cancer 55 : 191-195, 1987.
18. Preston-Martin S, Jin F, Duda MJ and Mack WJ: A case-control study of thyroid cancer in women under age 55 in Shanghai (People's Republic of China). Cancer Causes Control 4: 431-440, 1993.

19. Rossing MA, Voigt LF, Wicklund KG and Daling JR: Reproductive factors and risk of papillary thyroid cancer in women. Am J Epidemiol 151: 765-772, 2000.

20. Schonfeld SJ, Ron E, Kitahara CM, et al: Hormonal and reproductive factors and risk of postmenopausal thyroid cancer in the NIH-AARP Diet and Health Study. Cancer Epidemiol 35: e85-e90, 2011.

21. Takezaki T, Hirose $\mathrm{K}$, Inoue M, et al: Risk factors of thyroid cancer among women in Tokai, Japan. J Epidemiol 6: 140-147, 1996.

22. Truong T, Orsi L, Dubourdieu D, Rougier Y, Hemon D and Guenel P: Role of goiter and of menstrual and reproductive factors in thyroid cancer: a population-based case-control study in New Caledonia (South Pacific), a very high incidence area. Am J Epidemiol 161: 1056-1065, 2005.

23. Wingren G, Hatschek T and Axelson O: Determinants of papillary cancer of the thyroid. Am J Epidemiol 138: 482-491, 1993.

24. Yasmeen S, Cress R, Romano PS, et al: Thyroid cancer in pregnancy. Int J Gynaecol Obstet 91: 15-20, 2005.

25. Zivaljevic V, Vlajinac H, Jankovic R, et al: Case-control study of female thyroid cancer - menstrual, reproductive and hormonal factors. Eur J Cancer Prev 12: 63-66, 2003.

26. Yoshimura M, Nishikawa M, Yoshikawa N, et al: Mechanism of thyroid stimulation by human chorionic gonadotropin in sera of normal pregnant women. Acta Endocrinol (Copenh) 124: $173-178,1991$.

27. Kumar A, Klinge CM and Goldstein RE: Estradiol-induced proliferation of papillary and follicular thyroid cancer cells is mediated by estrogen receptors $\alpha$ and $\beta$. Int $\mathrm{J}$ Oncol 36: $1067-1080,2010$ 